\title{
On-Line Fault Detection Technique for Voltage Transformers
}

\author{
Ting Lei, Marco Faifer, Roberto Ottoboni, Sergio Toscani \\ DEIB, Politecnico di Milano \\ Piazza Leonardo da Vinci 32 - 20133 Milano ITALY
}

\begin{abstract}
Conventional voltage transformers (CVTs) are still widely used as transducers in the power network, thanks to their high reliability, insulation capability, low drift over time and temperature. Their rugged construction is very often used as justification for skipping periodical tests and calibrations, that require putting them off-line, thus avoiding a time-consuming and expensive procedure. For this reason, in the last years, a growing interest has been addressed towards the study of online diagnostic and calibration procedures. The typical approach is based on the frequency response analysis that permits, under sinusoidal conditions, to detect possible deterioration of the behavior of CVT. Anyway, the real interest is to check the CVT fleet already installed and operating on the grid without requiring their disconnection from the grid. As well known, distribution grid voltage features a non negligible harmonic distortion, which may allow the online evaluation of the frequency response of the transformer, by simply connecting a reference transducer. Unfortunately, being the harmonics much lower than the fundamental, this approach cannot be employed in a straightforward way because of the nonlinear behavior of the CVT. This paper proposes an innovative condition monitoring technique of CVTs based on a nonlinear simplified Volterra model. This opens the way to a new approach to the on-site characterization of CVTs, exploiting the actual voltage of the grid and thus not requiring its disconnection.
\end{abstract}

Keywords—voltage transformer; Volterra model; harmonic monitoring; frequency response; diagnostic.

\section{INTRODUCTION}

Nowadays, along with the concept of sustainable development, most renewable power generators, such as solar panels and wind turbines, are connected to the grid by means of switch-mode interface converters. On the other hand, many electrical loads use a power electronics input stage. Since these generators and converters are nonlinear devices, harmonic distortion in the grid has noticeably increased in the last years. Harmonics should be monitored since they may be responsible for increasing energy losses, overheating in power transformers and malfunction of electronic switches. Consequently, the transducers installed in the grid have to guarantee a proper measurement accuracy not only at the mains frequency, but also over a suitable frequency bandwidth.

Another mandatory prerequisite of the transducers is high reliability and negligible drift over time of their measurement accuracy. Any replacement and/or off-line calibration of these devices imply interruption of the grid service, thus having great economic impact.

Despite the availability of a new generation of non-conventional instrument transformers (NCITs), based on electronics, optical technologies, etc., the CIT, based on the law of induction, is still the most widely used device for current and voltage measurement in medium and high voltage systems. In a overall evaluation, their robustness, passive working principle and long-term stability are features very often preferred to the wider bandwidth, higher linearity and smaller size of NCITs.

As a consequence, the CVTs are also used for voltage harmonic monitoring. In order to guarantee proper performances, it is necessary to characterize their accuracy in the frequency band of interest. The conventional approach is to evaluate the ratio and the phase error versus frequency, by applying a sinusoidal frequency sweep. In the last years, many researches have been addressed to exploit the knowledge of CVT frequency response for improving the performances. In particular, the target was to extend and improve the bandwidth of the device with real-time frequency compensation techniques [1], 
[2], [3]. More interesting, the frequency response analysis (FRA) can be employed as a diagnostic tool for detecting fault conditions caused by geometrical or electrical changes within the transformer. In turn, the frequency response can be divided into several sub-bands, each of them allows detecting different transformer faults [4], [5], [6]. For example, in [6], the author summarizes that any variation of the frequency response within the range of up to $2 \mathrm{kHz}$ suggests a probable fault in the transformer winding, which are responsible for about $20 \%$ of all the transformer failures [8].

Despite their conceptual interest, this frequency-response based approach is not suitable for an online implementation. A CVT is intrinsically nonlinear and does not operate with a pure sinusoidal input, but rather with a complex signal. In real working conditions, intermodulation products between fundamental and harmonics plays a significant role in the input-output relationship [9]. Hence, it is clear that this method cannot be applied without putting the transformer offline and supplying its primary with a sine wave.

In literature, several on-line approaches for diagnostics purpose can be found. Most of them are based on dissolved gas analysis (DGA) [10], [11], [12], [13], [14]. The triangle method, fuzzy logic, support vector machine and artificial neural network have been used for detecting the fault condition based on the DGA results [10], [15], [16]. However, the DGA approach is complex and it can only be applied for oil-filled transformer. The diagnostic approaches derived from vibration signals [17], [18], [19] and acoustic signals [20], [21], [22] are still under investigation and they can be only applied to few fault conditions. Moreover, these techniques do not allow monitor the metrological characteristic of the analyzed system. Among the aforementioned methods, the only one that can be used for the metrological characterization is the FRA.

In fact, this fault detection technique compares the input-output relationship of a reference model with that of the actual system. It is clear that if the analyzed system is non-linear, the reference model cannot be a simple frequency response function, such as in the traditional FRA. In fact, the model error can jeopardize the diagnostic decision. Moreover, since the target is to use as test signal the distorted power line voltage (online diagnostic), intermodulation must be taken into account. Therefore a nonlinear model must be employed as reference. A possible solution is represented by the simplified frequency-domain Volterra model that the authors have proposed in a recent paper [23]. Volterra models are typically extremely complicated consisting in a huge number of coefficients. However, this number can be greatly reduced by exploiting the fact that electrical signals in AC power systems consists of a main fundamental component and harmonics that are much lower in amplitude.

The proposed method require to preliminarily identify the simplified Volterra model of the considered CVT. It permits to predict, with a good accuracy, its output for quasi-sinusoidal input voltages. By comparing the actual output with that predicted by the model fed with the same input voltage, the health state of the analyzed CVT can be assessed [24]. The chosen diagnostic index is the TVE (Total Vector Error) for each harmonic. In this work, an online fault detection technique based on this approach is presented and its capability to recognize inter-turn short circuits is experimentally evaluated. This approach can be used for in-field analysis of the health status and of the metrological characteristics of CVT.

The Volterra theory and the simplified model developed by the authors in previous work are briefly recalled in Section II and III. In Section IV, the proposed model has been validated by experimental tests. In Section V the proposed diagnostic approach is described while in section VI its experimental validation is provided.

\section{THE VOLTERRA MODEL}

The Volterra series is one of the most widely used methods for representing nonlinear systems. For a single-input, single-output (SISO), time-invariant system, the input/output representation based on the Volterra series is given by the equation (1) [25].

$$
\left\{\begin{array}{l}
y(t)=\sum_{i=1}^{\infty} y_{i}(t) \\
y_{i}(t)=\int_{-\infty}^{+\infty} \ldots \int_{-\infty}^{+\infty} h_{i}\left(\tau_{1}, \tau_{2}, \ldots, \tau_{i}\right) \prod_{l=1}^{i} u\left(t-\tau_{l}\right) d \tau_{1} \ldots d \tau_{i}
\end{array}\right.
$$

where $u(t)$ is the input signal, $y(t)$ the output, $y_{i}(t)$ the contribution to the output of the $i$-th order homogeneous subsystem 
characterized by its kernel $h_{i}$, which is the generalization of the impulse response of a linear time invariant system.

Equation (1) cannot be employed in actual applications since it is continuous time, other having an infinite number of kernels and unlimited memory length. Therefore, from a practical point of view, a more significant representation of a nonlinear system is the truncated, discrete Volterra series:

$$
\left\{\begin{array}{l}
y(n)=\sum_{i=1}^{I} y_{\mathrm{i}}(n) \\
y_{i}(n)=\sum_{k_{1}=1}^{K-1} \cdots \sum_{k_{i}=1}^{K-1} h_{i}\left(k_{1}, \ldots, k_{i}\right) \prod_{l=1}^{i} u\left(n-k_{l}\right)
\end{array}\right.
$$

where $I$ is the order of nonlinearity and $K$ is the memory length. It can be shown that the truncated Volterra model is characterized by a number of independent coefficients $c_{i n d}$, which rapidly grows with $I$ and $K$ [26]:

$$
c_{\text {ind }}=\sum_{i=1}^{I} \frac{(K+i-1) !}{(K-1) ! i !}
$$

Now, let us suppose that the input signal is periodic, characterized by the fundamental angular frequency $\omega_{0}$ and containing a set of harmonic components, being $N$ the highest order. In this case the model can be noticeably simplified by moving to the frequency domain. Let us call $U\left(j m \omega_{0}\right)=U(m)$ and $Y\left(j m \omega_{0}\right)=Y(m)$ the spectra of the input and output respectively. The frequency-domain expression of (2) becomes:

$$
\left\{\begin{array}{l}
Y(m)=\sum_{i=1}^{I} Y_{i}(m) \\
Y_{i}(m)=\sum_{-N \leq m_{1}, \ldots, m_{i} \leq N} H_{i}\left(m_{1}, \ldots, m_{i}\right) \prod_{l=1}^{i} U\left(m_{l}\right)
\end{array}\right.
$$

Where:

$$
\sum_{l=1}^{i} m_{l}=m
$$

(4) represents the relationship between the harmonic components of the input and output signals. The total number of independent coefficients now results:

$$
c_{\text {ind }}^{\prime}=\sum_{i=1}^{I} \frac{(M+i-1) !}{(M-1) ! i !}
$$

Basically, it is the same as (3) where $K$ is replaced by $M=2 N+1$; since $M$ is usually considerably lower than the memory length $K$, the frequency domain representation allows to considerably simplify the model. However, the number of coefficients still rapidly grows with $I$, therefore low nonlinearity orders are usually considered in practical applications.

\section{THE SIMPLIFIED VOLTERRA MODEL}

As previously discussed, in order to test the instrument transformers under realistic working conditions, the test voltage has to resemble those really present in the grid. In particular, the standard EN 50160 [27] defines the limits in terms of harmonics amplitude, as summarized in Table 1. 
Table 1: harmonics voltage limits according to EN 50160.

\begin{tabular}{|l|l|l|l|l|l|}
\hline \multicolumn{2}{|l|}{ Odd harmonics } & \multicolumn{2}{l|}{ Even harmonics } \\
\hline Not multiples of 3 & \multicolumn{2}{|l|}{ Multiples of 3 } & \multirow{2}{*}{ Order } & $\begin{array}{l}\text { Relative } \\
\text { Amplitude }\end{array}$ \\
\cline { 1 - 4 } Order & $\begin{array}{l}\text { Relative } \\
\text { amplitude }\end{array}$ & Order & $\begin{array}{l}\text { Relative } \\
\text { amplitude }\end{array}$ & & $2.0 \%$ \\
\hline 5 & $6.0 \%$ & 3 & $5.0 \%$ & 2 & $1.0 \%$ \\
\hline 7 & $5.0 \%$ & 9 & $1.5 \%$ & 4 & $0.5 \%$ \\
\hline 11 & $3.5 \%$ & 15 & $0.5 \%$ & $6 \ldots .24$ & \\
\hline 13 & $3.0 \%$ & 21 & $0.5 \%$ & & \\
\hline 17 & $2.0 \%$ & & & & \\
\hline 19 & $1.5 \%$ & & & & \\
\hline 23 & $1.5 \%$ & & & & \\
\hline 25 & $1.5 \%$ & & & & \\
\hline
\end{tabular}

Now, let us investigate the application of the frequency-domain Volterra approach to instrument transformers. According to (4), the $m$-th harmonic component of the output results from the contributions of $I$ nonlinear homogeneous subsystems. For each order $i$, the subsystem output is calculated by combining all the possible $i$-th order intermodulation products between the input harmonic components, weighted by the corresponding $i$-th order coefficient. If the input signal complies with Table 1, the amplitude of the largest harmonic component is only $6 \%$ of the fundamental frequency. For this reason, the contributions to the output of the intermodulation products involving more than one harmonic component are quite small. In other words, for each order $i$ the model can be noticeably simplified by considering only the intermodulation products consisting of the fundament frequency component and at most one harmonic component, namely those which can be written in the form:

$$
U(1)^{i_{p}} U(-1)^{i-i_{p}-1} U(n)
$$

being $0 \leq i_{p} \leq i$. This allows obtaining a simplified Volterra model, so that higher orders can be employed without having to estimate a huge number of coefficients during its identification. In fact, the maximum number of coefficients now reduces to:

$$
c_{\max }^{\prime \prime}=M \frac{I(I+1)}{2}=M L
$$

Furthermore, not all of them are independent: therefore the number of coefficients to be identified is actually lower than $c_{\text {max }}^{\prime \prime}$. However, even neglecting this, it is clear that (8) is much smaller than (6), thus the hypothesis about the input signal allows a drastic simplification of the model. The fifth order simplified model employed in this paper has been presented and deeply discussed in [23]; obviously only the independent coefficients have been considered in the model.

For each spectral component, the input-output relation of the simplified model can be written as:

$$
Y(m)=\mathbf{U}^{\text {red }}(m) \mathbf{H}^{\text {red }}(m)
$$

$\mathbf{U}^{\text {red }}(m)$ and $\mathbf{H}^{\text {red }}(m)$ are vectors having no more than $L$ elements. Now, the target is to identify the model: it corresponds to compute $\mathbf{H}^{\text {red }}(m)$ for the considered spectral components. This can be performed by applying a set of $R \geq L$ independent input signals to the system to be identified and measuring the responses. Then, it is possible to write:

$$
\mathbf{U}_{i d}^{\text {red }}(m)=\left[\begin{array}{c}
\mathbf{U}_{1}^{\text {red }}(m) \\
\mathbf{U}_{2}^{\text {red }}(m) \\
\vdots \\
\mathbf{U}_{R}^{\text {red }}(m)
\end{array}\right] \quad \mathbf{Y}_{i d}=\left[\begin{array}{c}
Y_{1}(m) \\
Y_{2}(m) \\
\vdots \\
Y_{R}(m)
\end{array}\right]
$$

Having employed a proper set of identification signals, the matrixes $\mathbf{U}_{i d}{ }^{\text {red }}(m)$ are full rank. The model coefficients can be estimated using the least squares approach. 


\section{EXPERIMENTAL VALIDATION OF THE PROPOSED MODEL}

The fifth order simplified Volterra model recalled in the previous section has been used to represent the behavior of a CVT under test (TUT) having 0.5 accuracy class, $200 \mathrm{~V} / 100 \mathrm{~V}$ voltage ratio and $20 \mathrm{VA}$ rated burden. Its primary winding has been connected to an arbitrary voltage generator, similar to that described in [28], able to generate the proper voltage signals required by the tests. Primary and secondary winding voltages of the TUT has been collected by using two highaccuracy resistive dividers. The dividers have been calibrated in the calibration lab at our Department, by means of a Fluke 8508A 8.5-digit multimeter. The linearity of the dividers, that is the critical parameter of the performed analysis, is much higher than that of the considered CVT. The basic structure and a pic of the experimental setup are shown in Fig. 1 .

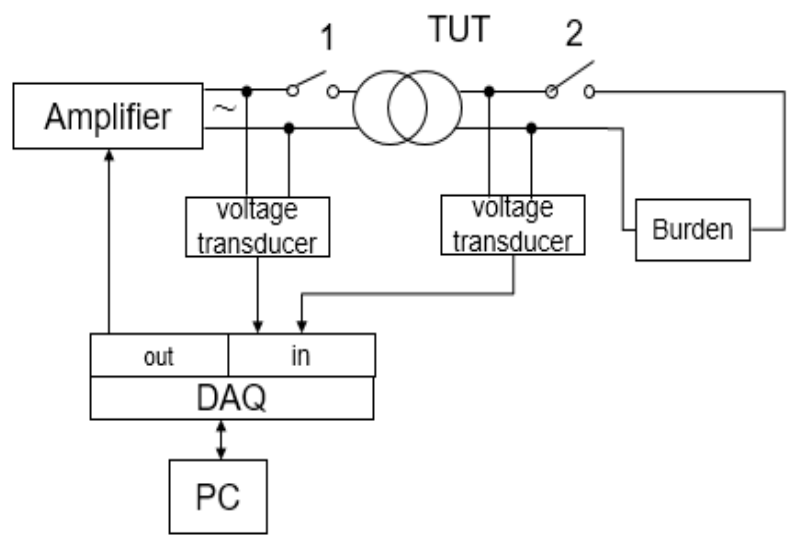

a)

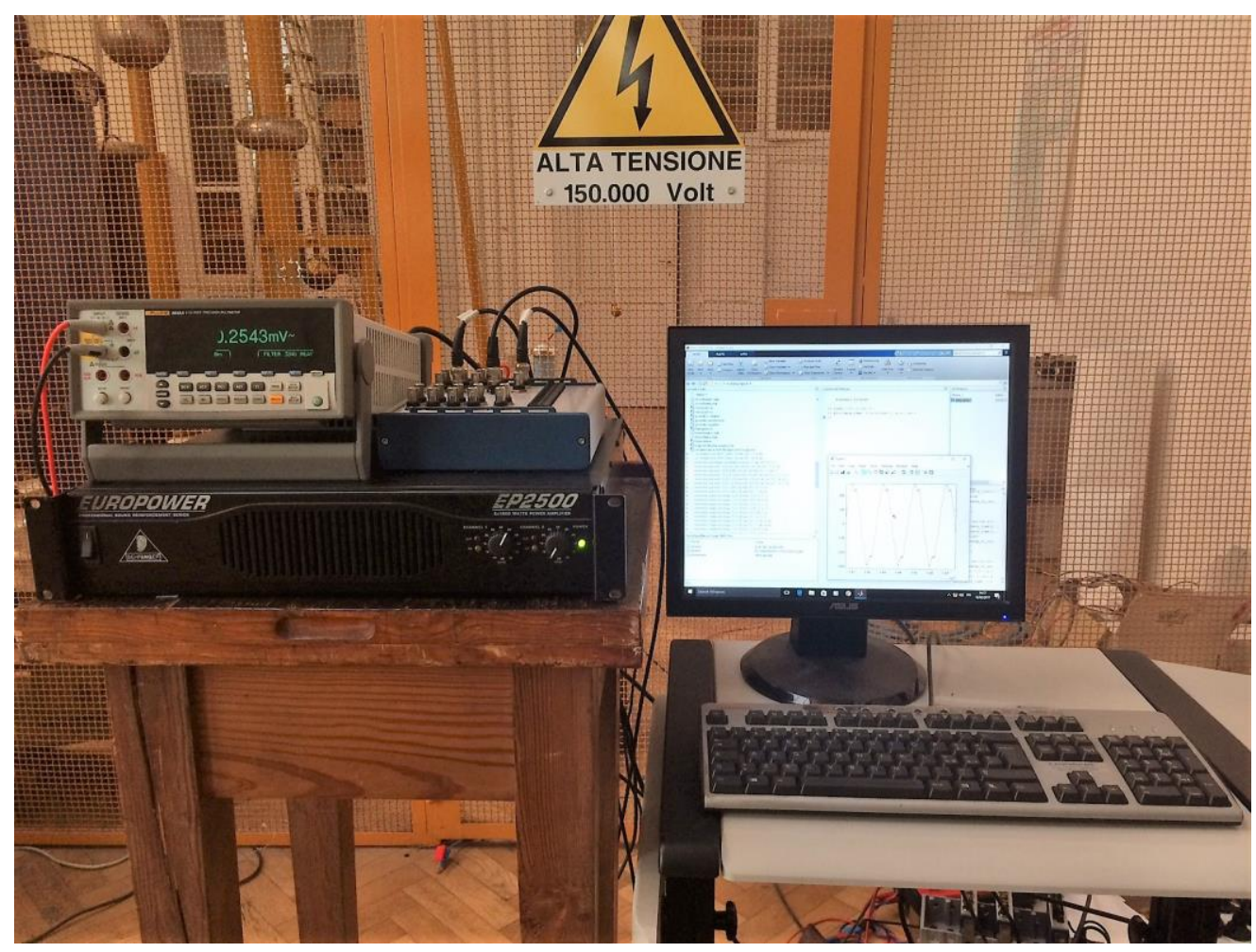

(b)

Fig. 1. Experimental setup: (a) schematic diagram (b) photo of devices 
The setup has been characterized by evaluating the type A measurement uncertainty $u$ of the TVE (Table 2). In order to do that, a multisine signal complying with the limits in Table 1 has been generated and measured 100 times with the system described in Fig. 1.

Table 2: Measurement uncertainty of the system.

\begin{tabular}{|c|c|c|c|c|c|c|c|c|c|c|}
\hline Frequency $[\mathrm{Hz}]$ & 50 & 100 & 150 & 200 & 250 & 300 & 350 & 400 & 450 & 500 \\
\hline$u(T V E)$ & $2.0 \times 10^{-3}$ & $1.4 \times 10^{-2}$ & $1.2 \times 10^{-2}$ & $1.1 \times 10^{-2}$ & $5.4 \times 10^{-3}$ & $3.2 \times 10^{-2}$ & $3.9 \times 10^{-2}$ & $4.4 \times 10^{-2}$ & $3.9 \times 10^{-2}$ & $5.6 \times 10^{-2}$ \\
\hline
\end{tabular}

First of all, the model of the TUT has been identified by estimating its coefficient as described in the previous section. The identification has been performed by using signals generated according to the condition Test 1 reported in Table 3 . Each signal is composed by a fundamental at $50 \mathrm{~Hz}$ plus some harmonics whose amplitudes and phases are randomly generated for each test signal, as specified in Table 3. The model has been then tested in order to verify its accuracy for different input signals. The validation process consisted of three different tests, whose conditions are summarized in Table 3. In Test 1 , harmonics have the same amplitude but different phases with respect to the identification process. In Test 2 , the harmonic amplitudes have been increased with respect to that of Test 1 . Finally, in Test 3, input signals complying with the harmonic component limits provided by EN 50160 have been used. Each test has been repeated 140 times.

Table 3: Test conditions.

\begin{tabular}{|c|c|c|c|c|c|}
\hline & \multirow[b]{3}{*}{$\begin{array}{l}\text { Number of } \\
\text { signals }\end{array}$} & \multicolumn{4}{|c|}{ Input signal } \\
\hline & & \multicolumn{2}{|c|}{ Fundamental } & \multicolumn{2}{|l|}{ 2nd - 10th harmonics } \\
\hline & & $\begin{array}{c}\text { Amplitude } \\
\text { (percentage of } \\
\text { the rated voltage) }\end{array}$ & Phase & $\begin{array}{c}\text { Amplitude } \\
\text { (percentage of fundamental component) }\end{array}$ & Phase \\
\hline Test 1 & 140 & $\begin{array}{l}\text { Random } \\
(90-110 \%)\end{array}$ & $\begin{array}{l}\text { Random } \\
\quad(0-\pi)\end{array}$ & $\begin{array}{c}\text { 2nd:1\%, 3rd:5\%, 4th:1\%, 5th:5\% } \\
\text { 6th:1\%, 7th:5\%, 8th:1\%, 9th:1.5\%, } \\
\text { 10th:1\% }\end{array}$ & Random \\
\hline Test 2 & 140 & $\begin{array}{l}\text { Random } \\
(90-110 \%)\end{array}$ & $\begin{array}{l}\text { Random } \\
\quad(0-\pi)\end{array}$ & $\begin{array}{c}\text { 2nd:2\%, 3rd:7\%, 4th:2\%, 5th:8\% } \\
\text { 6th:2\%, 7th:7\%, 8th:2\%, 9th:3\%, } \\
\text { 10th:2\% }\end{array}$ & Random \\
\hline Test 3 & 140 & $\begin{array}{c}\text { Random } \\
(90-110 \%)\end{array}$ & $\begin{array}{c}\text { Random } \\
(0-\pi)\end{array}$ & Random (under the limits of Table 1) & Random \\
\hline
\end{tabular}

All the signals have been applied by using the amplitude profile shown in Fig. 2 in order to reduce remanent flux in the TUT, thus improving the quality of the identification. The amplitude of the signal has been gradually increased from zero, maintained constant during the test, and then slowly decreased as shown in the Fig. 2. The signals have been acquired for $10 \mathrm{~s}$ with $100 \mathrm{kHz}$ sampling frequency by means of a simultaneous sampling DAQ board having 16-bit resolution (National Instruments NI6356).

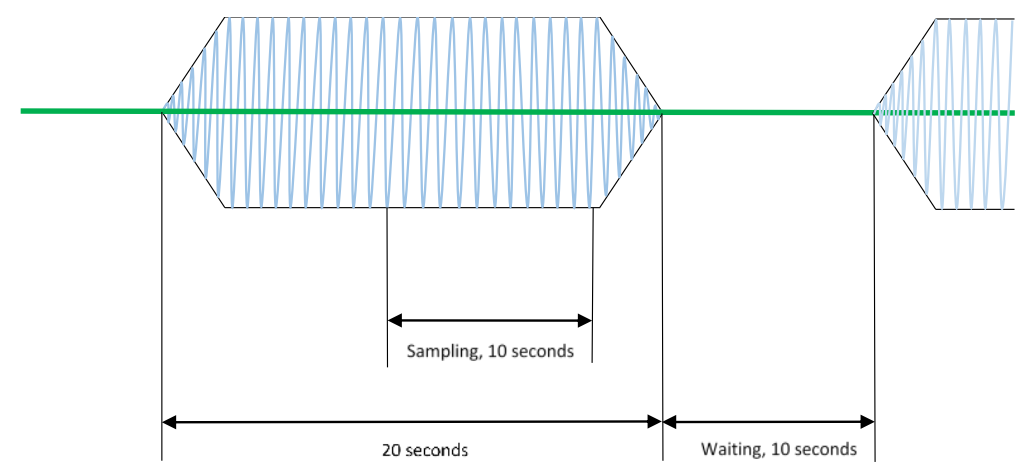

Fig. 2. Test procedure: scheme of the test voltage profile over time. 
In order to evaluate the difference between the physical output of the TUT and that of its model, several indexes have been evaluated, namely amplitude error $(A E)$, phase error $(P E)$ and total vector error $(T V E)$ :

$$
\begin{aligned}
& A E=\left|\frac{\left|Y_{M}(m)\right|-\left|Y_{A}(m)\right|}{\left|Y_{A}(m)\right|}\right| \times 100 \\
& P E=\left|\angle Y_{M}(m)-\angle Y_{A}(m)\right| \\
& T V E=\left|\frac{Y_{M}(m)-Y_{A}(m)}{Y_{A}(m)}\right| \times 100
\end{aligned}
$$

where $Y(m)$ represents the $m$-th order output harmonic. The subscripts $M$ and $A$ denote the model output and the actual output, respectively.

The results are reported in Table 4, Table 5 and Table 6, where, for each index, the 95th percentile of the parameter distributions $\left(A E_{95}, P E_{95}, T V E_{95}\right)$ are listed.

Table 4: Experimental results of Test 1 .

\begin{tabular}{|c|c|c|c|}
\hline TEST1 & $A E_{95}$ & $P E_{95}\left[{ }^{\circ}\right]$ & $T V E_{95}$ \\
\hline $50 \mathrm{~Hz}$ & $2.1 \times 10^{-2}$ & $7.0 \mathrm{E}-03$ & $2.4 \times 10^{-2}$ \\
\hline $100 \mathrm{~Hz}$ & $6.6 \times 10^{-2}$ & $3.7 \times 10^{-2}$ & $8.3 \times 10^{-2}$ \\
\hline $150 \mathrm{~Hz}$ & $7.3 \times 10^{-2}$ & $4.4 \times 10^{-2}$ & $9.4 \times 10^{-2}$ \\
\hline $200 \mathrm{~Hz}$ & $7.3 \times 10^{-2}$ & $4.6 \times 10^{-2}$ & $9.4 \times 10^{-2}$ \\
\hline $250 \mathrm{~Hz}$ & $8.2 \times 10^{-2}$ & $4.4 \times 10^{-2}$ & $9.5 \times 10^{-2}$ \\
\hline $300 \mathrm{~Hz}$ & $8.6 \times 10^{-2}$ & $5.1 \times 10^{-2}$ & $1.1 \times 10^{-1}$ \\
\hline $350 \mathrm{~Hz}$ & $1.2 \times 10^{-1}$ & $7.5 \times 10^{-2}$ & $1.6 \times 10^{-1}$ \\
\hline $400 \mathrm{~Hz}$ & $8.4 \times 10^{-2}$ & $4.6 \times 10^{-2}$ & $1.0 \times 10^{-1}$ \\
\hline $450 \mathrm{~Hz}$ & $2.3 \times 10^{-1}$ & $1.3 \times 10^{-1}$ & $2.7 \times 10^{-1}$ \\
\hline $500 \mathrm{~Hz}$ & $4.6 \times 10^{-2}$ & $2.5 \times 10^{-2}$ & $5.3 \times 10^{-2}$ \\
\hline
\end{tabular}

Table 5: Experimental results of Test 2.

\begin{tabular}{|c|c|c|c|}
\hline TEST2 & $A E_{95}$ & $P E_{95}\left[^{\circ}\right]$ & $T^{\circ} E_{95}$ \\
\hline $50 \mathrm{~Hz}$ & $1.6 \times 10^{-2}$ & $6.9 \mathrm{E}-03$ & $1.9 \times 10^{-2}$ \\
\hline $100 \mathrm{~Hz}$ & $7.1 \times 10^{-2}$ & $3.7 \times 10^{-2}$ & $8.2 \times 10^{-2}$ \\
\hline $150 \mathrm{~Hz}$ & $6.9 \times 10^{-2}$ & $4.2 \times 10^{-2}$ & $9.2 \times 10^{-2}$ \\
\hline $200 \mathrm{~Hz}$ & $7.6 \times 10^{-2}$ & $4.6 \times 10^{-2}$ & $9.4 \times 10^{-2}$ \\
\hline $250 \mathrm{~Hz}$ & $6.6 \times 10^{-2}$ & $4.1 \times 10^{-2}$ & $8.3 \times 10^{-2}$ \\
\hline $300 \mathrm{~Hz}$ & $7.4 \times 10^{-2}$ & $4.7 \times 10^{-2}$ & $1.0 \times 10^{-1}$ \\
\hline $350 \mathrm{~Hz}$ & $9.8 \times 10^{-2}$ & $5.7 \times 10^{-2}$ & $1.3 \times 10^{-1}$ \\
\hline $400 \mathrm{~Hz}$ & $8.6 \times 10^{-2}$ & $5.0 \times 10^{-2}$ & $1.1 \times 10^{-1}$ \\
\hline $450 \mathrm{~Hz}$ & $1.4 \times 10^{-1}$ & $8.2 \times 10^{-2}$ & $1.7 \times 10^{-1}$ \\
\hline $500 \mathrm{~Hz}$ & $4.7 \times 10^{-2}$ & $2.5 \times 10^{-2}$ & $5.4 \times 10^{-2}$ \\
\hline
\end{tabular}

Table 6: Experimental results of Test 3.

\begin{tabular}{|c|c|c|c|}
\hline TEST3 & $A E_{95}$ & $P E_{95}\left[^{\circ}\right]$ & $T^{\circ}$ \\
\hline $50 \mathrm{~Hz}$ & $2.7 \times 10^{-2}$ & $7.1 \times 10^{-3}$ & $2.9 \times 10^{-2}$ \\
\hline $100 \mathrm{~Hz}$ & $8.9 \times 10^{-2}$ & $4.5 \times 10^{-2}$ & $1.0 \times 10^{-1}$ \\
\hline $150 \mathrm{~Hz}$ & $8.8 \times 10^{-2}$ & $4.4 \times 10^{-2}$ & $1.0 \times 10^{-1}$ \\
\hline $200 \mathrm{~Hz}$ & $7.7 \times 10^{-2}$ & $5.2 \times 10^{-2}$ & $1.1 \times 10^{-1}$ \\
\hline $250 \mathrm{~Hz}$ & $8.7 \times 10^{-2}$ & $5.1 \times 10^{-2}$ & $1.1 \times 10^{-1}$ \\
\hline $300 \mathrm{~Hz}$ & $8.9 \times 10^{-2}$ & $4.8 \times 10^{-2}$ & $1.1 \times 10^{-1}$ \\
\hline $350 \mathrm{~Hz}$ & $1.7 \times 10^{-1}$ & $9.4 \times 10^{-2}$ & $2.0 \times 10^{-1}$ \\
\hline $400 \mathrm{~Hz}$ & $9.0 \times 10^{-2}$ & $5.0 \times 10^{-2}$ & $1.1 \times 10^{-1}$ \\
\hline $450 \mathrm{~Hz}$ & $3.0 \times 10^{-1}$ & $1.8 \times 10^{-1}$ & $3.5 \times 10^{-1}$ \\
\hline $500 \mathrm{~Hz}$ & $4.8 \times 10^{-2}$ & $2.5 \times 10^{-2}$ & $6.0 \times 10^{-2}$ \\
\hline
\end{tabular}

It should be noticed that amplitude and phase errors are always well below the measurement errors corresponding to the accuracy class of the TUT, thus proving the accuracy of the model. For the sake of completeness, the frequency response (FR) of the TUT has been evaluated by using a swept sine wave input signal, and employed to predict the output 
of Test 3. Table 7 reports the comparison between the results obtained in this case and those given by the proposed nonlinear model.

Table 7. Comparison between nonlinear model and FR.

\begin{tabular}{|c|c|c|c|c|c|c|}
\hline \multirow{2}{*}{ TEST3 } & \multicolumn{2}{|c|}{$A E_{95}$} & \multicolumn{2}{c|}{$P E_{95}\left[{ }^{\circ}\right]$} & \multicolumn{2}{c|}{$T V E_{95}$} \\
\cline { 2 - 7 } & Model & FR & Model & FR & Model & FR \\
\hline $50 \mathrm{~Hz}$ & $2.7 \times 10^{-2}$ & $2.2 \times 10^{-2}$ & $7.1 \times 10^{-3}$ & $6.0 \mathrm{E}-03$ & $2.9 \times 10^{-2}$ & $2.4 \times 10^{-2}$ \\
\hline $100 \mathrm{~Hz}$ & $8.9 \times 10^{-2}$ & $8.8 \times 10^{-2}$ & $4.5 \times 10^{-2}$ & $4.2 \times 10^{-2}$ & $1.0 \times 10^{-1}$ & $1.0 \times 10^{-1}$ \\
\hline $150 \mathrm{~Hz}$ & $8.8 \times 10^{-2}$ & 1.3 & $4.4 \times 10^{-2}$ & $7.8 \times 10^{-1}$ & $1.0 \times 10^{-1}$ & 1.7 \\
\hline $200 \mathrm{~Hz}$ & $7.7 \times 10^{-2}$ & $1.4 \times 10^{-1}$ & $5.2 \times 10^{-2}$ & $8.9 \times 10^{-2}$ & $1.1 \times 10^{-1}$ & $1.8 \times 10^{-1}$ \\
\hline $250 \mathrm{~Hz}$ & $8.7 \times 10^{-2}$ & $4.0 \times 10^{-1}$ & $5.1 \times 10^{-2}$ & $2.3 \times 10^{-1}$ & $1.1 \times 10^{-1}$ & $5.1 \times 10^{-1}$ \\
\hline $300 \mathrm{~Hz}$ & $8.9 \times 10^{-2}$ & $1.4 \times 10^{-1}$ & $4.8 \times 10^{-2}$ & $8.3 \times 10^{-2}$ & $1.1 \times 10^{-1}$ & $1.8 \times 10^{-1}$ \\
\hline $350 \mathrm{~Hz}$ & $1.7 \times 10^{-1}$ & $2.0 \times 10^{-1}$ & $9.4 \times 10^{-2}$ & $1.2 \times 10^{-1}$ & $2.0 \times 10^{-1}$ & $2.5 \times 10^{-1}$ \\
\hline $400 \mathrm{~Hz}$ & $9.0 \times 10^{-2}$ & $1.3 \times 10^{-1}$ & $5.0 \times 10^{-2}$ & $8.0 \times 10^{-2}$ & $1.1 \times 10^{-1}$ & $1.7 \times 10^{-1}$ \\
\hline $450 \mathrm{~Hz}$ & $3.0 \times 10^{-1}$ & $4.8 \times 10^{-1}$ & $1.8 \times 10^{-1}$ & $2.8 \times 10^{-1}$ & $3.5 \times 10^{-1}$ & $5.8 \times 10^{-1}$ \\
\hline $500 \mathrm{~Hz}$ & $4.8 \times 10^{-2}$ & $1.1 \times 10^{-1}$ & $2.5 \times 10^{-2}$ & $5.3 \times 10^{-2}$ & $6.0 \times 10^{-2}$ & $1.4 \times 10^{-1}$ \\
\hline
\end{tabular}

As clearly pointed out, the linear model heavily fails at $150 \mathrm{~Hz}$, which is heavily affected by nonlinearities due to the iron core. On the contrary, these effect is instead well represented by the proposed nonlinear model. As a consequence, the simplified Volterra model can be employed as reference for online diagnostic procedures. In fact, once the input signal is known, any significant difference between the output signal of the CVT and that given by the model is an indicator of a possible change in the device behavior.

\section{THE DIAGNOSTIC PROCEDURE}

One of the main problems of conventional diagnostic techniques for CVTs is the need to disconnect them from the grid. In fact, if diagnostic methods like Sweep Frequency Response Analysis (SFRA) [29], transfer function analysis (TFA), wavelet analysis, etc. have to be implemented, it is necessary to excite the system with proper signals. All these methods are based on the idea that when some degradation phenomena occur in the device, its input-output response changes. Therefore by comparing the actual response with that expected when the system is considered healthy, it is possible to detect the degradation.

The idea here proposed is to use the simplified Volterra model of the TUT as reference for the comparison. As shown before, the Volterra model can be identified by using input voltage signals similar to those found in distribution networks. The diagnostic procedure require to connect a reference transducer in parallel to the TUT (Fig. 3) thus allowing an accurate measurement of the line voltage. By means of the TUT model identified in healthy condition $(H)$, (for example during the VT calibration or the first installation) it is possible to predict the secondary voltage of the TUT, and to evaluate the TVE between the actual and the predicted voltage. A fault is detected when the TVE is likely not compatible with the model accuracy. This can be performed in two ways. A first simple approach (Fig. 4) requires to compare the single value of TVE with the 95th percentile of the TVE characteristic of the TUT in healthy condition (TVE $95 \mathrm{H})$. It is clear that this approach is not very robust. A more accurate evaluation can be performed by considering the distribution of TVE evaluated with different input voltages (i.e.: monitoring the transformer output and input for a suitable period of time), and evaluating the 95th percentile, namely $T V E_{95}$. The comparison between $T V E_{95}$ and $T V E_{95 \mathrm{H}}$ is a more robust diagnostic indicator. 


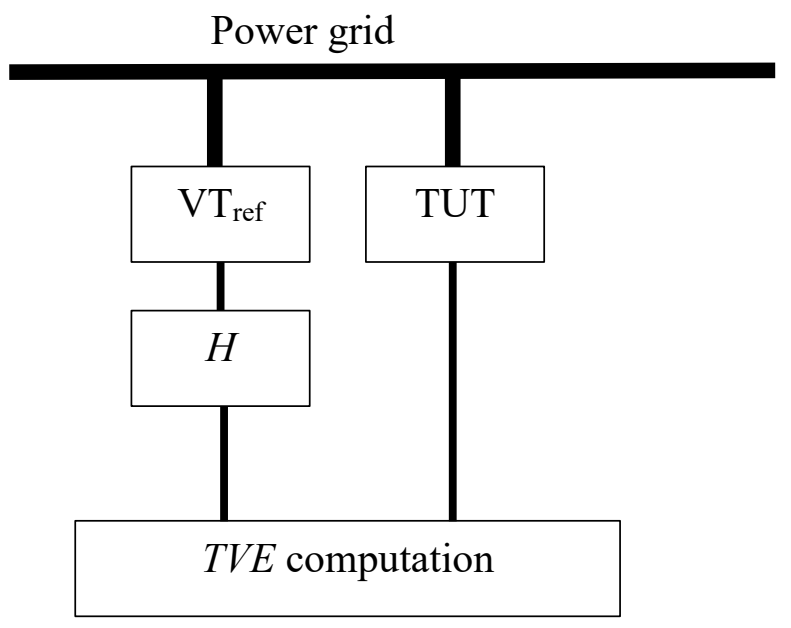

Fig. 3. Diagnostic approach.

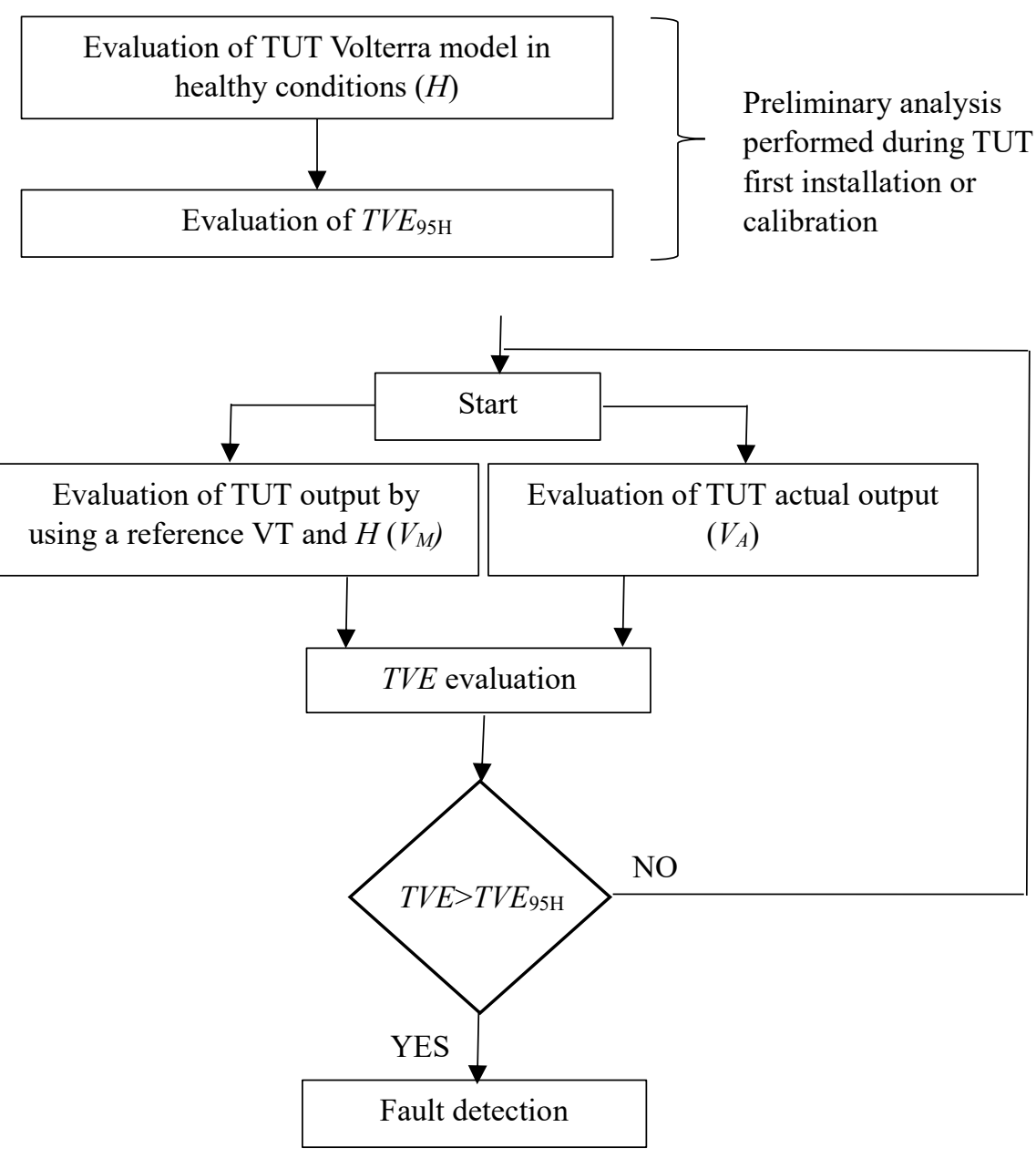

Fig. 4. Diagnostic procedure based on a single TVE comparison. 


\section{EXPERIMENTAL VALIDATION OF THE DIAGNOSTIC APPROACH}

In this section, the experimental activity devoted to prove the applicability of the proposed approach has been discussed. As aforementioned, many faults can occur in transformers such as inter-turn winding fault, insulation degradation between windings or versus ground, winding displacement [5]. For the experimental validation, the inter-turn winding fault has been considered. This fault can be simulated by means of multi-tap transformer and a resistor connected as shown in Fig. 5. In particular, the resistor has been connected between two auxiliary taps of the secondary winding of the transformer under test. This permits to emulate an inter-turn fault, in which the isolation between nearby turns has degraded. By changing the value of the resistance, it is possible to change the fault severity [8], [29], [30].

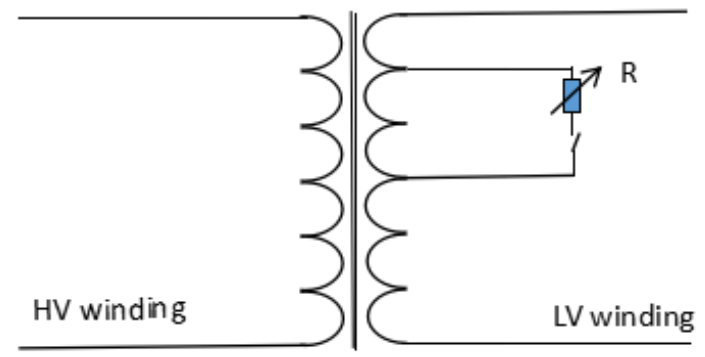

Fig. 5. Schematic diagram of a transformer, which has a variable resistor connected across secondary taps.

The primary winding has been fed with the signal already used in Test 3 and three values of resistance $R$ have been connected ( $1 \Omega, 2 \Omega$ and $4 \Omega$ ). The excitation signals have spectral components that vary both in phase and in amplitude so as to simulate repeated measurement performed on the grid. For each of the 140 runs of the test, two TVEs have been computed: one by using the Volterra model and the other by using the FR (by applying (12)). The obtained results have been reported in Table 8 and Table 9 where the 95th percentile values of the obtained distributions have been reported.

Table 8: 95th percentile of the TVE distribution obtained by using FR model.

\begin{tabular}{|c|c|c|c|c|}
\hline & $\mathrm{NR}$ & $4 \Omega$ & $2 \Omega$ & $1 \Omega$ \\
\hline $50 \mathrm{~Hz}$ & $8.4 \times 10^{-2}$ & $1.1 \times 10^{-1}$ & $1.5 \times 10^{-1}$ & $2.1 \times 10^{-1}$ \\
\hline $100 \mathrm{~Hz}$ & $4.3 \times 10^{-1}$ & $4.3 \times 10^{-1}$ & $4.4 \times 10^{-1}$ & $5.5 \times 10^{-1}$ \\
\hline $150 \mathrm{~Hz}$ & 6.4 & 6.3 & 6.3 & 6.0 \\
\hline $200 \mathrm{~Hz}$ & $4.1 \times 10^{-1}$ & $4.1 \times 10^{-1}$ & $5.4 \times 10^{-1}$ & $7.1 \times 10^{-1}$ \\
\hline $250 \mathrm{~Hz}$ & 1.6 & 1.6 & 1.6 & 1.7 \\
\hline $300 \mathrm{~Hz}$ & $4.6 \times 10^{-1}$ & $4.8 \times 10^{-1}$ & $6.3 \times 10^{-1}$ & $9.1 \times 10^{-1}$ \\
\hline $350 \mathrm{~Hz}$ & $7.3 \times 10^{-1}$ & $7.4 \times 10^{-1}$ & $9.3 \times 10^{-1}$ & 1.2 \\
\hline $400 \mathrm{~Hz}$ & $5.6 \times 10^{-1}$ & $6.3 \times 10^{-1}$ & $8.4 \times 10^{-1}$ & 1.2 \\
\hline $450 \mathrm{~Hz}$ & 1.2 & 1.2 & 1.3 & 1.7 \\
\hline $500 \mathrm{~Hz}$ & $6.9 \times 10^{-1}$ & $7.4 \times 10^{-1}$ & $9.6 \times 10^{-1}$ & 1.4 \\
\hline
\end{tabular}

Table 9: 95th percentile of the TVE distribution obtained by using Volterra model.

\begin{tabular}{|l|c|c|c|c|}
\hline & $\mathrm{NR}$ & $4 \Omega$ & $2 \Omega$ & $1 \Omega$ \\
\hline $50 \mathrm{~Hz}$ & $8.5 \times 10^{-2}$ & $1.1 \times 10^{-1}$ & $1.5 \times 10^{-1}$ & $2.1 \times 10^{-1}$ \\
\hline $100 \mathrm{~Hz}$ & $1.9 \times 10^{-1}$ & $2.0 \times 10^{-1}$ & $2.6 \times 10^{-1}$ & $3.5 \times 10^{-1}$ \\
\hline $150 \mathrm{~Hz}$ & $4.0 \times 10^{-1}$ & $3.8 \times 10^{-1}$ & $4.6 \times 10^{-1}$ & $5.8 \times 10^{-1}$ \\
\hline $200 \mathrm{~Hz}$ & $3.1 \times 10^{-1}$ & $3.4 \times 10^{-1}$ & $4.6 \times 10^{-1}$ & $6.8 \times 10^{-1}$ \\
\hline $250 \mathrm{~Hz}$ & $5.0 \times 10^{-1}$ & $5.4 \times 10^{-1}$ & $6.5 \times 10^{-1}$ & $9.3 \times 10^{-1}$ \\
\hline $300 \mathrm{~Hz}$ & $4.3 \times 10^{-1}$ & $4.6 \times 10^{-1}$ & $6.3 \times 10^{-1}$ & $8.7 \times 10^{-1}$ \\
\hline $350 \mathrm{~Hz}$ & $6.6 \times 10^{-1}$ & $6.7 \times 10^{-1}$ & $8.6 \times 10^{-1}$ & 1.2 \\
\hline $400 \mathrm{~Hz}$ & $5.5 \times 10^{-1}$ & $6.4 \times 10^{-1}$ & $8.3 \times 10^{-1}$ & 1.2 \\
\hline $450 \mathrm{~Hz}$ & $9.9 \times 10^{-1}$ & $9.5 \times 10^{-1}$ & 1.2 & 1.5 \\
\hline $500 \mathrm{~Hz}$ & $6.8 \times 10^{-1}$ & $7.4 \times 10^{-1}$ & $9.6 \times 10^{-1}$ & 1.5 \\
\hline
\end{tabular}

In order to show the capability of the proposed approach in detecting the faults, in Fig. 6, Fig. 7 and Fig. 8 the percentages of fault detection with the two models have been reported. These percentages have been computed by considering as threshold of detection the 95th percentile TVE of the no fault condition for both models. These percentages 
represent the probability of fault detection by considering a single measurement. It can be noticed that the technique based on Volterra model features much better performance.

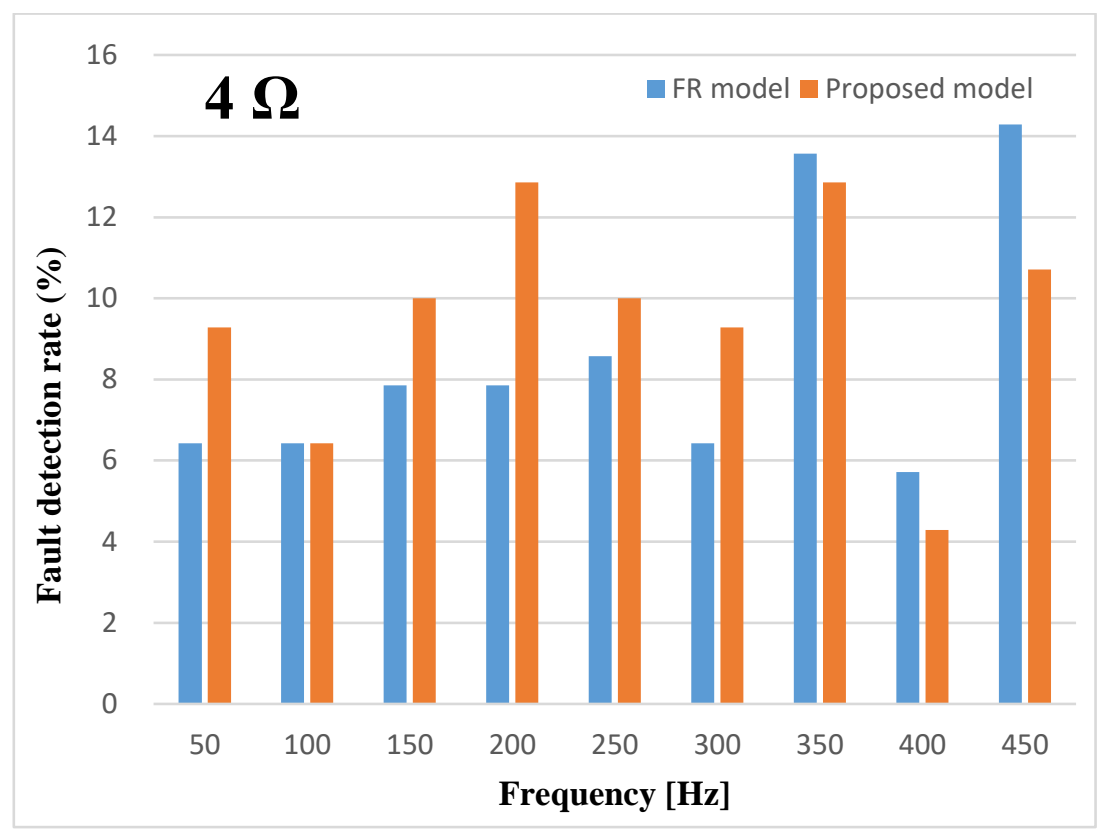

Fig. 6. Percentage of the successful fault detection with a fault resistance of $4 \Omega$.

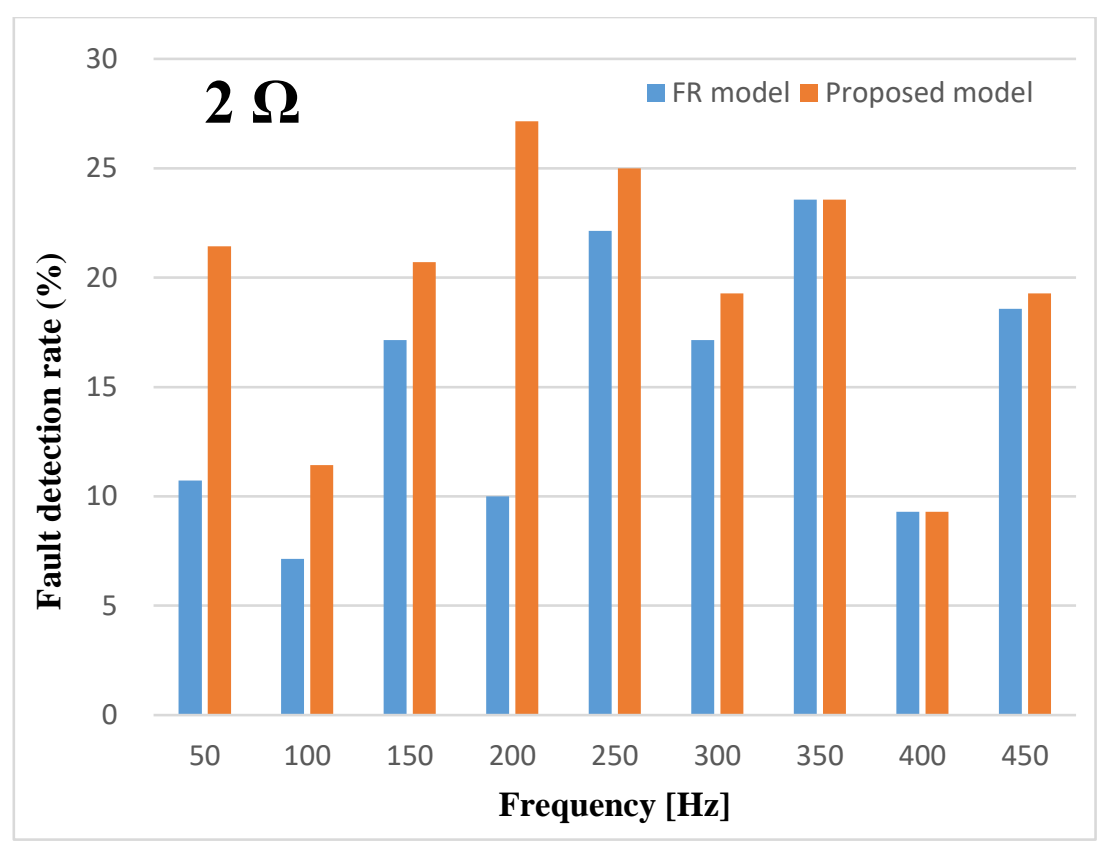

Fig. 7. Percentage of the successful fault detection with a fault resistance of $2 \Omega$. 


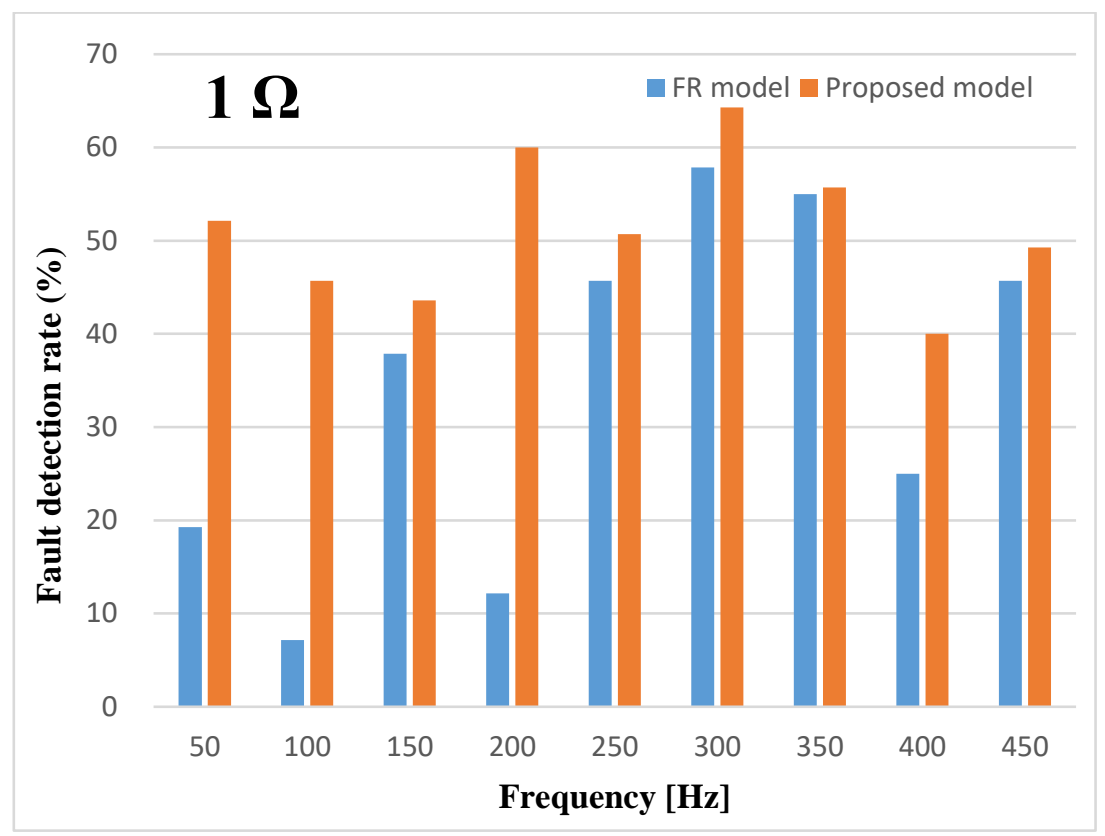

Fig. 8. Percentage of the successful fault detection with a fault resistance of $1 \Omega$.

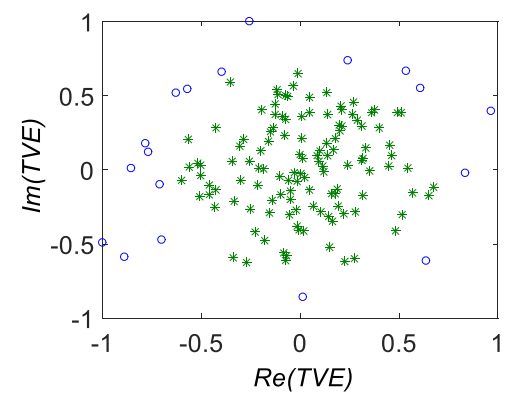

a)

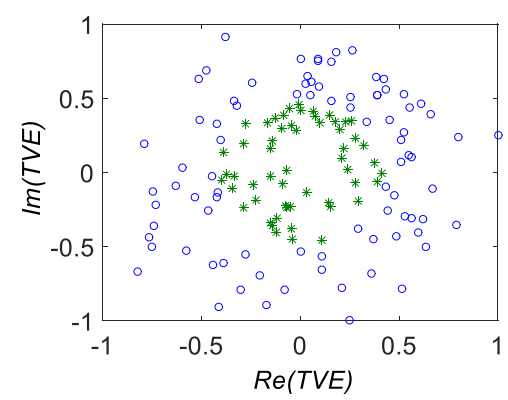

d)

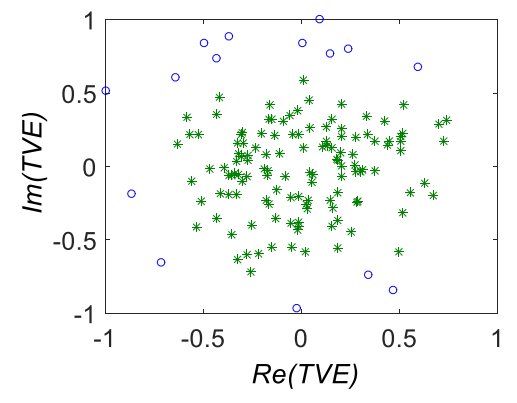

b)

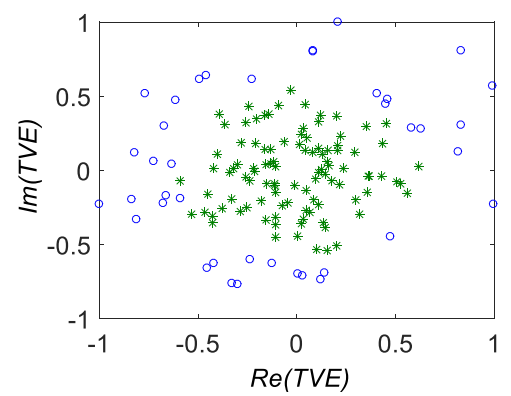

e)

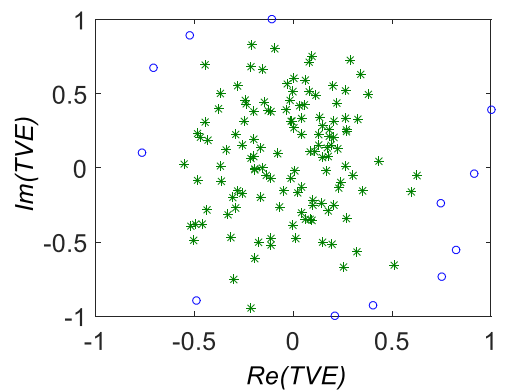

c)

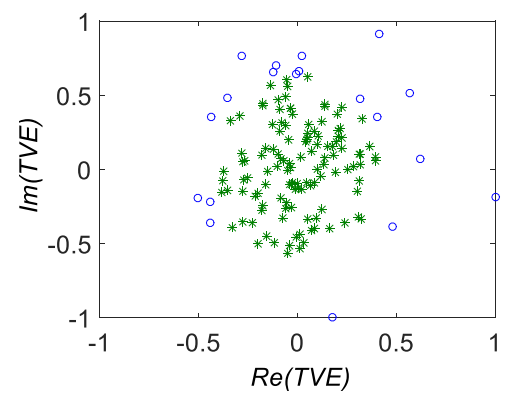

f)

Fig. 9. TVE estimation at $250 \mathrm{~Hz}$ using the FR (upper row) and proposed model (lower row) under different conditions; $1 \mathrm{ohm}(a, d), 2 \mathrm{ohm}(b, e)$, and $4 \mathrm{ohm}(c, f)$; the circles and stars in the figure represent fault and normal condition, respectively. 
In Fig. 9 the estimation of TVEs in terms of the real and imaginary component, normalized to the maximum value, at $250 \mathrm{~Hz}$ is reported. From the figure it can be noticed the better performances are achieved by using the nonlinear model.

By analyzing the distribution of the obtained results, it can be noticed that when the FR model is considered, it is not possible to detect the fault with a good reliability. In fact, the fault results in an increase of the TVE that is not considerably higher that the accuracy of the FR model. This is confirmed also by the analysis in Fig. 10 where the differences between the 95th percentiles of the TVE in case of fault and in healthy condition are reported.

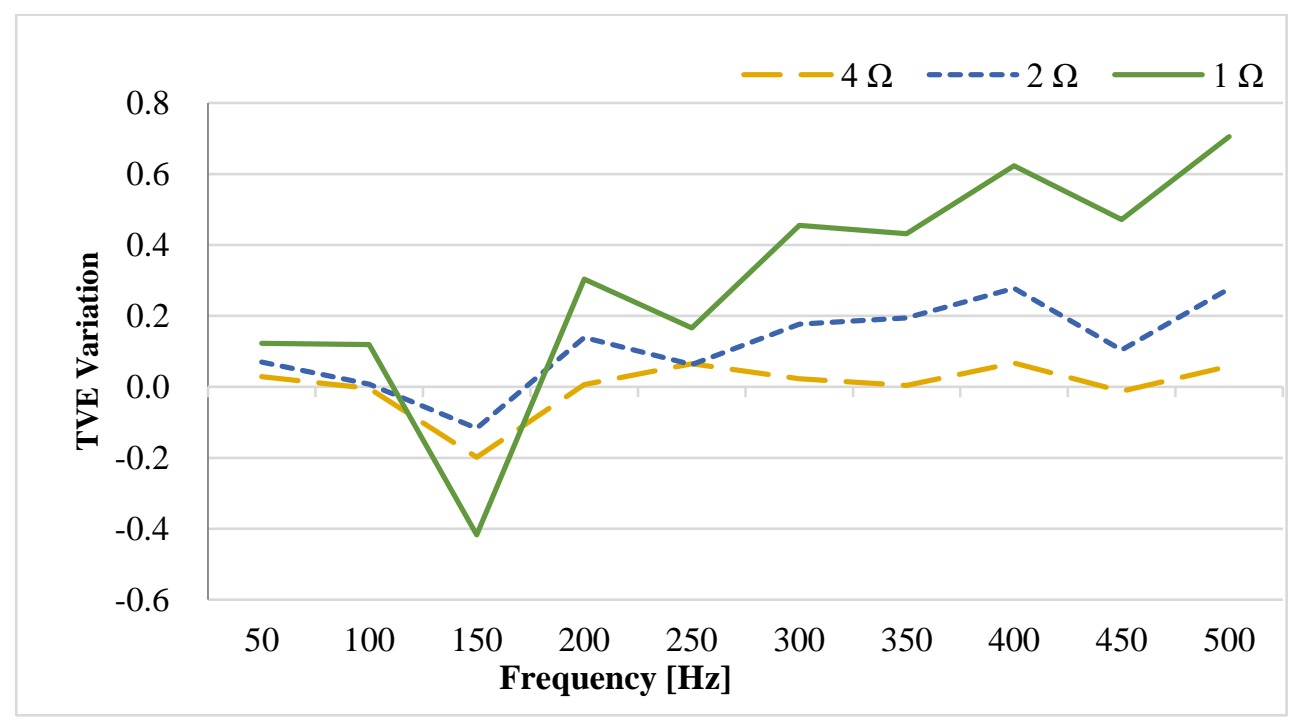

Fig. 10. Differences between the 95th percentile values in case of fault and no fault (FR model).

The same analysis has been performed with the data obtained by using the Volterra model. As it can be seen in Fig. 11 the fault can be identified as an increase of the TVE at every frequency. Moreover it can be seen that the TVE also allows to estimate the fault severity.

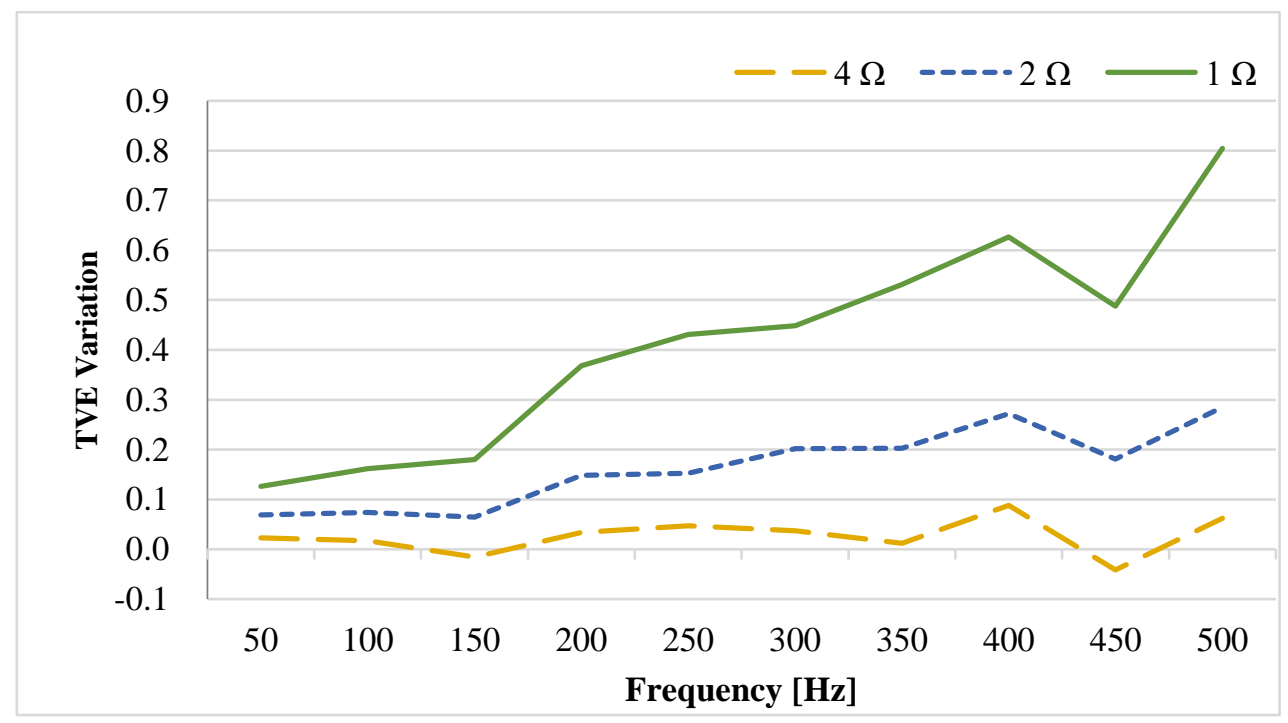

Fig. 11. Differences between the 95th percentile values in case of fault and no fault (nonlinear model). 


\section{CONCLUSION}

In this paper, a simplified frequency-domain Volterra model of a VT is used as reference for implementing a diagnostic technique. It has been shown that the model accuracy allows to detect changes in the electrical behavior of the VT without requiring the use of ad-hoc stimulus signal. In fact the proposed approach allows an online monitoring of the VT by exploiting the grid voltage. It has been shown that this approach features sensitivity much higher than that obtained by using the common frequency response analysis in detecting inter-turn faults. On the base of the results presented in this paper, our future research will focus on the evaluation of the method applicability for other kinds of faults.

\section{REFERENCES}

[1] M. Klatt, J. Meyer, M. Elst, P. Schegner, Frequency Responses of MV voltage transformers in the range of $50 \mathrm{~Hz}$ to $10 \mathrm{kHz}$, in Proc. 14th International Conference on Harmonics and Quality of Power - ICHQP 2010, 2010, pp. 1 -6.

[2] M. Kaczmarek, Development and application of the differential voltage to single-ended voltage converter to determine the composite error of voltage transformers and dividers for transformation of sinusoidal and distorted voltages, Measurement. 101 (2017) 53-61.

[3] A. E. Emanuel, Measurement of harmonics emitted by low voltage equipment: Testing requirements, in Proc. IEEE Power Eng. Soc. Winter Meeting, 1999, pp. 1115-1125.

[4] Santo Dolce, Edoardo Fiorucci, Giovanni Bucci, Flavio D'Innocenzo, Fabrizio Ciancetta, Antonio Di Pasquale, Test instrument for the automatic compliance check of cast resin insulated windings for power transformers, Measurement. 100 (2017) 50-61.

[5] J.R. Secue, E. Mombello, Sweep frequency response analysis (SFRA) for the assessment of winding displacements and deformation in power transformers, Electric Power Systems Research. 78 (2008) 1119-1128.

[6] Kennedy, G. M., A. J. McGrail, and J. A. Lapworth, Transformer sweep frequency response analysis (SFRA), Energize. (2007): 28-33.

[7] Mahdi Khanali, Amir Hayati-Soloot, Hans Kristian Høidalen, Shesha Jayaram, Study on locating transformer internal faults using sweep frequency response analysis, Electric Power Systems Research. 145 (2017) 55-62.

[8] Wang, M., A. J. Vandermaar, and K. D_ Srivastava , Review of condition assessment of power transformers in service, IEEE Electrical Insulation Magazine. 18 (2002) 12-25.

[9] T. Lei, L. Cristaldi, M. Faifer, R. Ottoboni, S. Toscani, C. Cherbaucich, P. Mazza, Behavior of Voltage Transformers under Distorted Conditions, in Proc. I2MTC16 - IEEE Instrumentation and Measurement Technology Conference, 2016, pp. 1-6.

[10] Ghoneim, Sherif SM, and Ibrahim BM Taha, A new approach of DGA interpretation technique for transformer fault diagnosis, International Journal of Electrical Power \& Energy Systems. 81 (2016) 265-274.

[11] Beykverdi, Mehrdad, and F. Faghihi, A new approach for transformer incipient fault diagnosis based on dissolved gas analysis (DGA), Nova Journal of Engineering and Applied Sciences. 3 (2016).

[12] Fernando Cortez Sica, Frederico Gadelha Guimarães, Ricardo de Oliveira Duarte, Agnaldo J.R. Reis, A cognitive system for fault prognosis in power transformers, Electric Power Systems Research. 127, (2015) 109-117.

[13] Khan, Shakeb A., Md Danish Equbal, and Tarikul Islam, A comprehensive comparative study of DGA based transformer fault diagnosis using fuzzy logic and ANFIS models, IEEE Transactions on Dielectrics and Electrical Insulation. 22 (2015) 590-596.

[14] De Faria, Haroldo, João Gabriel Spir Costa, and Jose Luis Mejia Olivas, A review of monitoring methods for predictive maintenance of electric power transformers based on dissolved gas analysis, Renewable and Sustainable Energy Reviews. 46 (2015) 201-209.

[15] Li, Jinzhong, et al., Optimal dissolved gas ratios selected by genetic algorithm for power transformer fault diagnosis based on support vector machine, IEEE Transactions on Dielectrics and Electrical Insulation. 23 (2016) 1198-1206.

[16] Hussein, Ahmed Raisan, M. M. Yaacob, and M. F. Othman, ANN expert system for diagnosing faults and assessing the quality insulation oil of power transformer depending on the DGA method, Journal of Theoretical and Applied Information Technology. 78 (2015) 278-285.

[17] Hong, Kaixing, et al., A method of real-time fault diagnosis for power transformers based on vibration analysis, Measurement Science and Technology. 26 (2015) 1-11.

[18] Duan, Ruochen, and Fenghua Wang, Fault Diagnosis of On-Load Tap-Changer in Converter Transformer Based on Time-Frequency Vibration Analysis, IEEE Transactions on Industrial Electronics- 63 (2016) 3815-3823.

[19] Kaixing Hong, Hai Huang, Yaqiong Fu, Jianping Zhou, A vibration measurement system for health monitoring of power transformers, Measurement. 93 (2016) 135-147.

[20] Tenbohlen, Stefan, et al., Diagnostic measurements for power transformers, Energies. 9 (2016) 1-25.

[21] Glowacz, Adam, Fault diagnostics of acoustic signals of loaded synchronous motor using SMOFS-25-EXPANDED and selected classifiers, Tehnički vjesnik. 23 (2016) 1365-1372.

[22] Glowacz, Adam, and Zygfryd Glowacz, Diagnostics of stator faults of the single-phase induction motor using thermal images, MoASoS and selected classifiers, Measurement. 93 (2016) 86-93.

[23] M. Faifer, R. Ottoboni, M. Prioli, S. Toscani, Simplified Modeling and Identification of Nonlinear Systems under Quasi-Sinusoidal Conditions, IEEE Transactions on Instrumentation and Measurement. 65 (2016) 1508-1515.

[24] Lei, T., Faifer, M., Ottoboni, R., Toscani, S., Towards a novel approach to the on-line diagnosis of the instrument transformer, 14th IMEKO TC10 Workshop on Technical Diagnostics 2016: New Perspectives in Measurements, Tools and Techniques for Systems Reliability, Maintainability and Safety, (2016), pp. 423-428.

[25] W J. Rugh, Nonlinear system theory. Baltimore: Johns Hopkins University Press, 1981.

[26] V. J. Mathews and G. L. Sicuranza, Polynomial Signal Processing (Wiley series in Telecommunications and Signal Processing). New York, NY, USA: Wiley, 2000.

[27] CENELEC, EN. "50160: Voltage characteristics of electricity supplied by public electricity networks," 2011. 
[28] M. Faifer, R. Ottoboni, S. Toscani, C. Cherbaucich, P. Mazza, Metrological Characterization of a Signal Generator for the Testing of MediumVoltage Measurement Transducers, IEEE Transactions on Instrumentation and Measurement. 64 (2015) 1837 -1846.

[29] Vahid Behjat, Abolfazl Vahedi, Alireza Setayeshmehr, Hossein Borsi, Ernst Gockenbach, Sweep frequency response analysis for diagnosis of low level short circuit faults on the windings of power transformers: An experimental study, International Journal of Electrical Power \& Energy Systems. 42 (2012) 78-90.

[30] M. Kezunovic and Yong Guo, Modeling and simulation of the power transformer faults and related protective relay behavior, IEEE Transactions on Power Delivery. 15 (2000) 44-50. 